\title{
Ground state energies from converging and diverging power series expansions
}

\author{
C. Lisowski, S. Norris, R. Pelphrey, E. Stefanovich, Q. Su and R. Grobe \\ Intense Laser Physics Theory Unit and Department of Physics \\ Illinois State University, Normal, IL 61790-4560 USA
}

It is often assumed that bound states of quantum mechanical systems are intrinsically non-perturbative in nature and therefore any power series expansion methods should be inapplicable to predict the energies for attractive potentials. However, if the spatial domain of the Schrödinger Hamiltonian for attractive one-dimensional potentials is confined to a finite length $\mathrm{L}$, the usual Rayleigh-Schrödinger perturbation theory can converge rapidly and is perfectly accurate in the weak-binding region where the ground state's spatial extension is larger than L. Once the binding strength is so strong that the ground state's extension is less than L, the power expansion becomes divergent, consistent with the expectation that bound states are non-perturbative. However, we propose a new truncated Borel-like summation technique that can recover the correct bound state energy from the diverging sum. We also show that perturbation theory becomes divergent in the vicinity of an avoided-level crossing. Here the same numerical summation technique can be applied to reproduce the correct finite energies from the diverging perturbative sums. 


\section{Introduction}

Perturbation theory was developed to analyze otherwise intractable problems in nearly all areas of science [1]. This method assumes that the full solution to a problem can be expressed as a power series expansion in terms of a (usually small) dimensionless parameter, which measures the strength of the interaction with the unperturbed system [2]. It is generally believed to be applicable when the solution is adiabatically connected to the unperturbed solution, i.e., it evolves smoothly from an unperturbed state. It is also assumed to fail for those scenarios where the perturbation transitions the system to a different phase with qualitatively different properties. In many important situations the dimensionless parameter is larger than the actual radius of convergence of the power series expansion and as a result the value of the series can no longer be computed by simply approximating the infinite series by its partial sums. However, numerous summation techniques [3] have been developed that permit us to accurately determine the (usually finite) value of these diverging sums.

In this work we examine the applicability of perturbation theory to predict the occurrence of quantum mechanical bound states in attractive short-range potentials in one spatial dimension. Here the dimensionless parameter is directly proportional to the strength of the interaction energy denoted by $V_{0}$. It is argued that the transition of a non-Hilbert state [with a wave function proportional to $\exp (\mathrm{i} \mathrm{k})$ for small momentum $\mathrm{k}$ ] to a normalizable discrete bound state (as $\mathrm{V}_{0}$ is increased from $\mathrm{V}_{0}=0$ ) is intrinsically non-perturbative. However, if a bound energy eigenstate is obtained numerically by diagonalizing the fully coupled Hamiltonian matrix in a finite basis set there is no ab initio reason why this transition from the quasi-continuum states to bound states should not be perturbative. This study was motivated by the well-known computational and also conceptual difficulty to obtain bound state energies from quantum field theoretical descriptions that often rely on perturbative approaches. In this work we also examine the applicability of perturbation theory to describe the quantum mechanical energies close to an avoided crossing [4-6]. This rather universal phenomenon occurs when two energetically neighboring energy states approach each other as the perturbation strength $V_{0}$ is increased. In the general case when there is no underlying symmetry (associated with a conserved quantity) the two levels repel each other and therefore avoid any degeneracy. As the character of the two involved state changes drastically after this passage through an avoided crossing, one might also wonder whether this "phase changing" transition can be computed based on perturbation theory.

While there exists obviously a large amount of literature in mathematics and physics about various summation techniques for diverging sums and their respective regions of applicability, almost all of these 
works assume that (a) the series have infinitely many terms and (b) that the precise form of each individual coefficient in the power series expansion is known analytically. In the opposite and purely numerical situation, where each coefficient has to be constructed computationally and therefore only a finite number of terms is accessible, much less is known. For example, we believe that the current work describes two important aspects for quantum mechanical bound states that have not been discussed before. First, we show that under a spatial confinement the perturbative method can actually be applied to predict reliable bound state energies. Second, in the corresponding diverging domain we propose a new approach that permits us to obtain the correct energies even if only a limited number of perturbative power series terms are available.

The article is structured as follows. In Section 2 we examine the regime where the perturbation $\mathrm{V}_{0}$ is sufficiently weak such that the spatial extension of the lowest energetic state is comparable to the finite size L of our numerical box. Here perturbation theory of arbitrarily high order is in fact convergent and highly accurate. We then show that for larger $\mathrm{V}_{0}$ the power expansion becomes divergent but this is not a problem as a proposed Borel-type summation technique [7,8] can recover the bound state energies. In Section 3 we examine the energies before and after an avoided crossing. Before the avoided crossing the series is convergent and therefore can be summed up using the finite partial sums whereas after the avoided crossing the perturbative expansion becomes divergent. However, we show that also here the finite value of this divergent series can be obtained by the Borel summation technique and its prediction matches perfectly the exact one. We propose a new numerical truncated Borel-based technique that permits us to estimate the diverging sums from only a finite number of perturbative terms. We finish this article with an outlook to future challenges.

\section{Converging and diverging perturbation theory for bound states}

While our main study focuses on the Schrödinger Hamiltonian $H\left(V_{0}\right)=p^{2} /(2 m)+V(x)$, where we use the short range one dimensional Yukawa potential $V(x)=-V_{0} \exp (-b|x|)$, we have also examined a finite-range and a zero-range potential. We use atomic units such that $m=\hbar=1$. In order to obtain accurate eigenvalues and eigenstates we have represented the Hamiltonian by a finite matrix. As basis states we have chosen momentum eigenstates as well as (for a better comparison) eigenstates of the position operator confined to a numerical box of extension L [9]. As a second (purely numerical) parameter we have introduced D, which is the total number of basis states. Details of these resulting $\mathrm{D} \times \mathrm{D}$ Hamiltonian matrices can be found in Appendix A. We also assumed periodic boundaries for all of our basis states. While the 
parameter D was chosen sufficiently large to have complete numerical convergence, the energies associated with those states whose spatial extension exceeds L do naturally depend on the size of L. The numerically determined eigenvalues were compared with those obtained with perturbation theory of up to $64^{\text {th }}$-order. The details for the perturbative calculations are summarized in Appendix B.

We should note that for an infinitely extended system (corresponding to $\mathrm{L} \rightarrow \infty$ ) all bound state energies for the attractive Yukawa potential $\mathrm{V}(\mathrm{x})=-\mathrm{V}_{0} \exp (-\mathrm{b}|\mathrm{x}|)$ can be found in a semi-analytical form [10], $\mathrm{E}_{\text {bound }}$ $=-b^{2} v_{0}^{2} /(8 m)$, where $v_{0}$ is the order of the Bessel function of first kind $J(v ; z)$ with argument $\mathrm{z} \equiv$ $\left(8 \mathrm{~V}_{0 \mathrm{~m}}\right)^{1 / 2} / \mathrm{b}$. For states that are even in $\mathrm{x}$ ( such as the ground state) the order $v_{0}$ is computed from the condition involving the derivative $\mathrm{d} J\left(v_{0} ; \mathrm{z}\right) / \mathrm{dz}=0$. Very similarly, the corresponding dimensionless parameter $v \square$ for the energies of the odd parity states needs to be calculated from $J(v ; z)=0$. In Appendix B we provide more details and show that for small strength of the potential, i.e., $8 \mathrm{~V}_{0} \mathrm{~m} / \mathrm{b}^{2}<<1$, the ground state energy grows quadratically with the potential strength, $E_{g}=-\left(2 \mathrm{~m} / \mathrm{b}^{2}\right) \mathrm{V}_{0}{ }^{2}$.

\subsection{Converging perturbative expansion for the bound state energy}

In order to show that there are two distinctly different regions, we begin this section by examining the spatial profile of the lowest energetic eigenstate first. In Figure 1 we show the spatial probability density of the eigenstate of $\mathrm{H}\left(\mathrm{V}_{0}\right)$ with the lowest energy for four potential strengths $\mathrm{V}_{0}$.

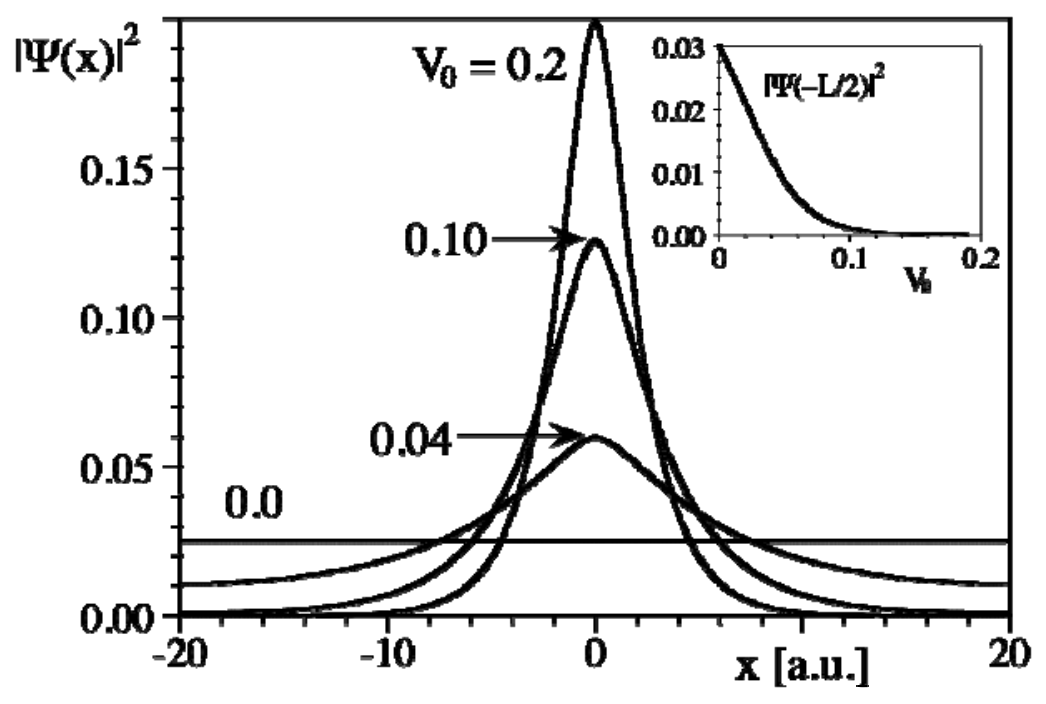


Figure 1 The spatial probability density of the lowest energetic energy eigenstate of the Hamiltonian with the attractive Yukawa potential $\mathrm{V}(\mathrm{x})=-\mathrm{V}_{0} \exp (-|\mathrm{x}|)$ for four potential strengths $\mathrm{V}_{0}$. The data were obtained by diagonalizing the Hamiltonian matrix in its spatial representation, with the numerical parameters $D=200$ and numerical grid spacing $\Delta x=0.2$. In the inset we display the value of the density at the left edge $\mathrm{x}=-\mathrm{L} / 2$ of the box as a function of $\mathrm{V}_{0}$.

The ground state evolves smoothly from the spatially delocalized lowest energetic continuum state $\Psi(\mathrm{x})=\mathrm{L}^{1 / 2}$ for $\mathrm{V}_{0}=0$, which has a vanishing unperturbed energy $\mathrm{E}=0$ and is normalized as $\int_{-\mathrm{L} / 2}^{\mathrm{L} / 2}|\Psi(\mathrm{x})|^{2} \mathrm{dx}$ $=1$. It is clear that for small values of $\mathrm{V}_{0}(<0.08$ a.u. $)$ the ground state's spatial extension is equal to the size $\mathrm{L}$ of the numerical box, which we chose as $\mathrm{L}=40$ a.u. in our figure. In the inset of the figure we have displayed the value of the density at the left edge of the box $x=-20$ a.u. as a function of $V_{0}$. Once the strength $\mathrm{V}_{0}$ exceeds the value close to $\mathrm{V}_{0}=\mathrm{V}_{\text {crit }}=0.08$ a.u., the width of the ground state $\Psi(\mathrm{x})$ begins to decrease from $\mathrm{L}$. We therefore would expect that in the very weak coupling regime $\mathrm{V}_{0}<\mathrm{V}_{\text {crit }}$ the spectrum should be a function of $\mathrm{L}$, whereas for $\mathrm{V}_{0}>\mathrm{V}_{\text {crit }}$ the energy of the ground state should no longer depend on $\mathrm{L}$. This expectation is confirmed in Figure 2, where we have graphed the ground state energy for two constant values of $\mathrm{V}_{0}$ as a function of $\mathrm{L}$.

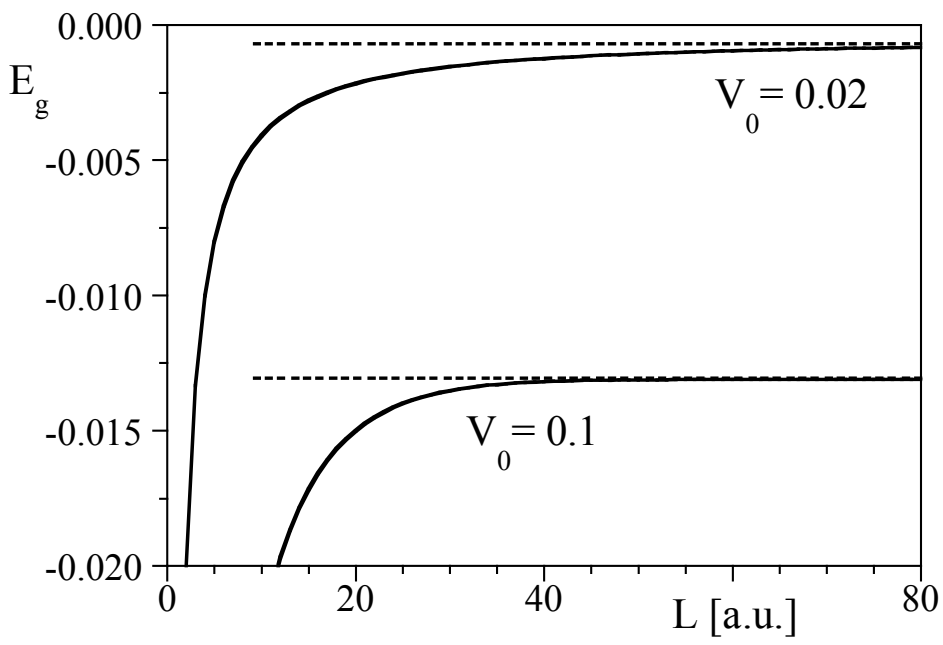

Figure 2 The ground state energy for two fixed potential strengths $\mathrm{V}_{0}$ as a function of the size of our numerical box L. The dashed lines are the two corresponding semi-analytical values $\left(-7 \times 1.710^{-4}\right.$ a.u. and $-1.31 \times 10^{-2}$ a.u.) for the ground state energy, corresponding to $L \rightarrow \infty$. [D=200 numerical grid points]

The data also show that for $\mathrm{V}_{0}=0.1$ a.u. and $\mathrm{L}>40$ a.u. the ground state energy approaches the semi-analytical limit $E_{g}=-b^{2} v_{0}^{2} /(8 \mathrm{~m})$, (which is $E_{g}=-0.013$ a.u.) as derived in Appendix A. 
We should note that the critical value $\mathrm{V}_{\text {crit }}$ decreases with increasing box size $\mathrm{L}$. The Rayleigh-Schrödinger perturbative prediction for the ground state energy is given by the power series expansion

$$
\mathrm{Eg}\left(\mathrm{V}_{0}\right)=\Sigma_{\mathrm{n}=0}^{\infty} \mathrm{e}_{\mathrm{n}} \mathrm{V}_{0}^{\mathrm{n}}
$$

The unperturbed energy $(\mathrm{n}=0)$ vanishes, $\mathrm{e}_{0}=0$, associated with the momentum eigenstate with zero momentum. In order to examine the validity of perturbation theory in the regime for $\mathrm{V}_{0}<\mathrm{V}_{\text {crit, }}$, we have graphed in Figure 3 the percent error of the partial sum $\mathrm{S}(\mathrm{N}) \equiv \Sigma_{\mathrm{n}=0} \mathrm{~N}_{\mathrm{n}} \mathrm{V}_{0}{ }^{\mathrm{n}}$ compared to the exact ground state energy $E_{\text {exact}}$, obtained from the numerical diagonalization of the Hamiltonian matrix. It is defined as

$$
\% \text { Error(N) } \equiv 100\left|\Sigma_{n=0}{ }^{N} e_{n} V_{0}^{n}-E_{\text {exact }}\right| /\left|E_{\text {exact }}\right|
$$
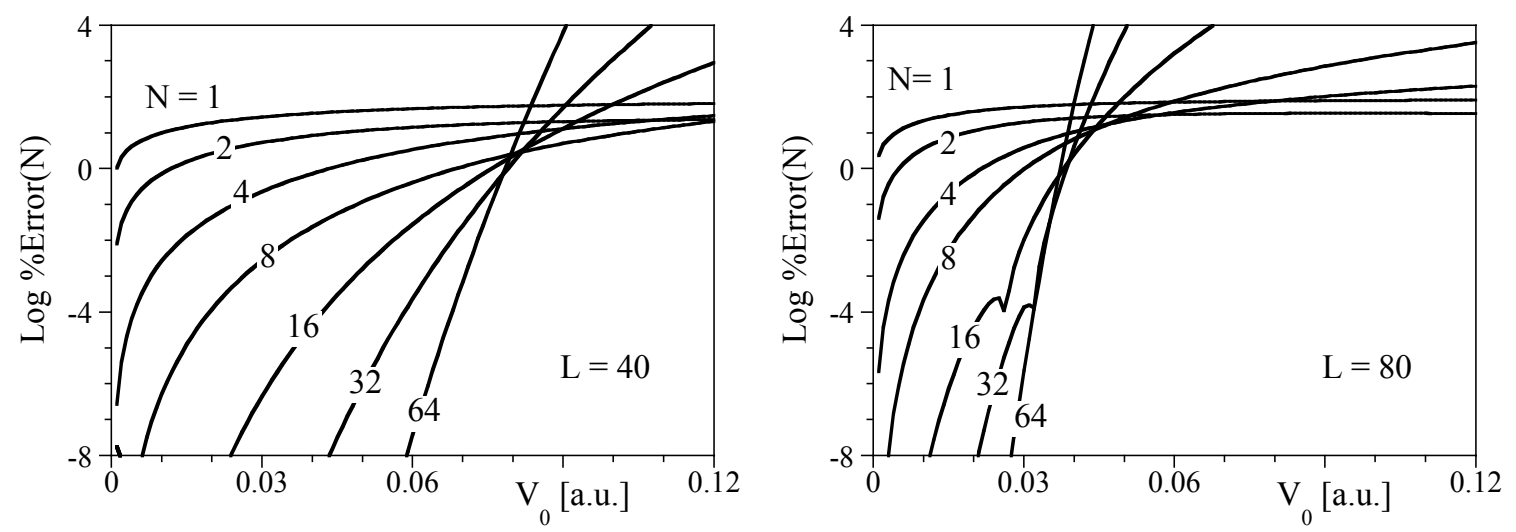

Figure 3 The percent error for approximating the ground state energy of the Yukawa potential by $\mathrm{N}$-th order perturbation theory as a function of $\mathrm{V}_{0}$ for a numerical box $\mathrm{L}=40$ a.u. and $\mathrm{L}=80$ a.u. $[\mathrm{D}=201]$

We see in the left Figure 3 that for small values of $\mathrm{V}_{0}<0.08$ a.u. the perturbation theory up to $64^{\text {th }}$-order converges very rapidly. For example, for $\mathrm{V}_{0}=0.06$ a.u. the exact ground state energy is $\mathrm{E}_{\mathrm{g}}=5.417022906 \times 10^{-3}$ a.u. and truncating the perturbative series after only 64 terms leads to an error of less than $10^{-8} \%$. On the other hand, we also see that the error for each partial sum decreases as $\mathrm{V}_{0}$ is increased. This decrease (reflected in the rate of convergence) is, of course, expected for any perturbative power series expansion. At about $\mathrm{V}_{0}=0.08$ a.u., the series becomes divergent as the crossings of the higher-order corrections of the curves clearly suggest. For $\mathrm{V}_{0}>0.08$ a.u., the more terms we keep in our 
expansion, the worse is the total error due to perturbation theory. We note that this divergent regime agrees roughly with the regime discussed in Figure $2\left(\mathrm{~V}_{\text {crit }}<\mathrm{V}_{0}\right)$ for which the ground state energy no longer depends on L.

For comparison, we have displayed in the right Figure 3 the same percent error, but for a larger numerical box $\mathrm{L}=80$ a.u.. We see that in the perturbative regime the overall percent error is comparable to that of the smaller box $\mathrm{L}=40$ a.u. However, for large $\mathrm{V}_{0}$ many more higher-order terms in the perturbative expansion are required to obtain the same accuracy. In fact, for $\mathrm{V}_{0}$ close to 0.04 a.u. we note that the perturbative series becomes divergent. This apparent scaling suggests that the radius of convergence of the perturbation theory in $\mathrm{V}_{0}$ might be inversely proportional to $\mathrm{L}$. Below we will examine this conjecture more quantitatively.

This divergent behavior as $\mathrm{L}$ is increased is not so unexpected if we analyze the lowest-order partial sums in more detail. The first-order correction is simply given by the matrix element $\mathrm{e}_{1} \mathrm{~V}_{0}{ }^{1}=\langle\mathrm{k}=0|\mathrm{~V}| \mathrm{k}=0\rangle$ $=\langle\mathrm{V}\rangle=\int \mathrm{dx} \mathrm{L} \mathrm{L}^{-1 / 2} \mathrm{~V}(\mathrm{x}) \mathrm{L}^{-1 / 2}$, where the integration limits extend from $-\mathrm{L} / 2$ to $\mathrm{L} / 2$. We find that as $\mathrm{V}(\mathrm{x})$ is short-ranged the term $\langle\mathrm{V}\rangle$ vanishes in the limit $\mathrm{L} \rightarrow \infty$. In other words, the energy decreases linearly in $\mathrm{V}_{0}$ only for a finite numerical box.

In appendix $\mathrm{B}$ we derived that the second-order term $\mathrm{e}_{2} \mathrm{~V}_{0}{ }^{2}$ is given by $\langle\mathrm{VGV}\rangle$, where $\mathrm{G}$ denotes the corresponding truncated resolvent containing the operator $1 /\left(0-\mathrm{p}^{2} / 2 \mathrm{~m}\right)$. In appendix $\mathrm{D}$, we show that the second-order term does not depend on L. In fact, it is quite reasonable to assume that all expansion coefficients $e_{n}$ scale like $L^{n-2}$ as a function of the box size $L$. This leads us to conjecture that the perturbative expansion can be rewritten as

$$
\mathrm{E}_{\mathrm{g}}\left(\mathrm{V}_{0}\right)=\mathrm{L}^{-2} \Sigma_{\mathrm{n}=0}^{\infty} \alpha_{\mathrm{n}}\left(\mathrm{L} \mathrm{V}_{0}\right)^{\mathrm{n}}
$$

where the new effective expansion coefficients $\alpha_{n}$ depend only very weakly on L. In order to examine this conjecture we have graphed in Figure 4 the first seven coefficients $\alpha_{\mathrm{n}}$ 


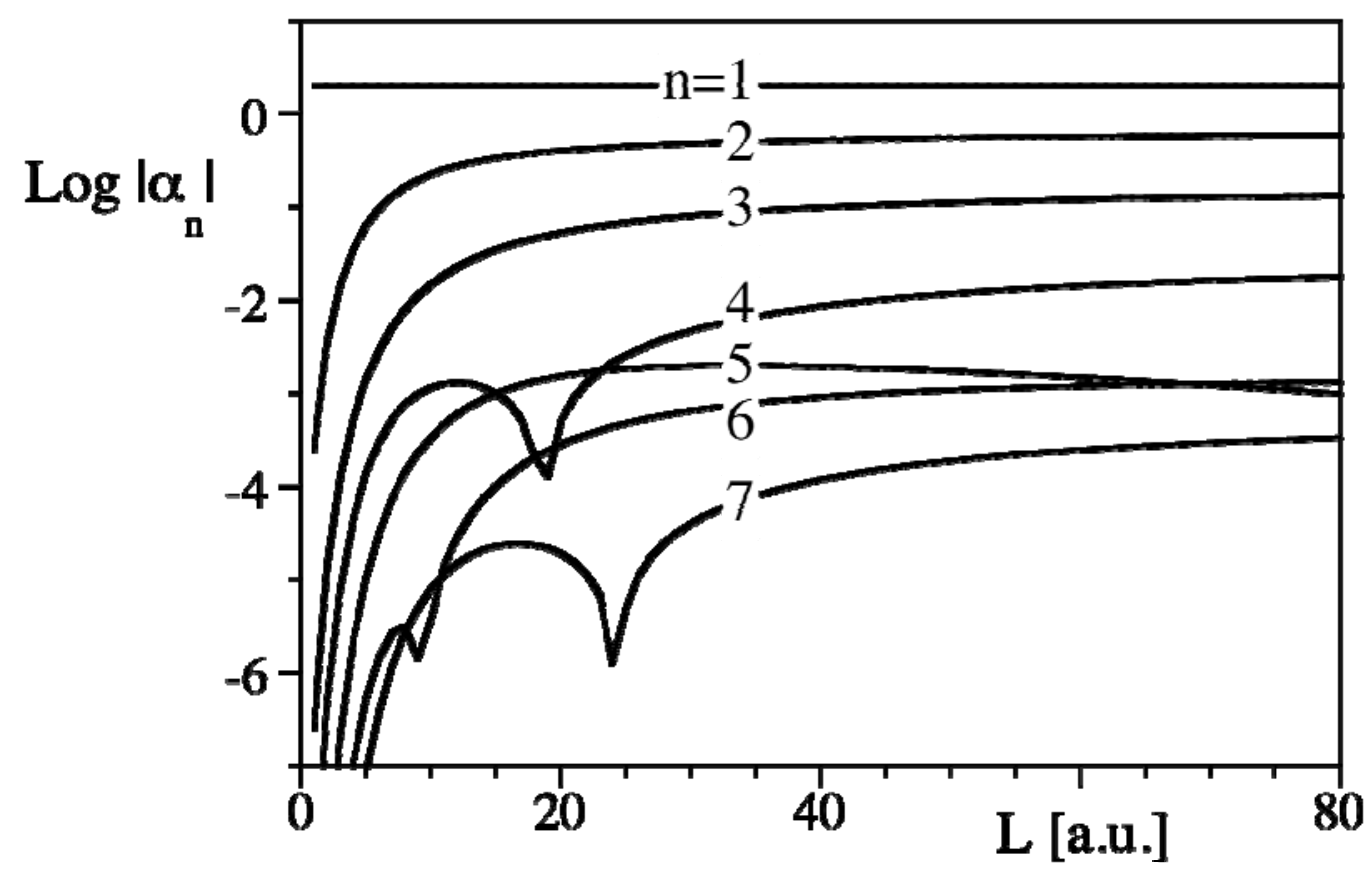

Figure 4 The effective perturbative coefficients $\alpha_{n}$ as a function of the order $n$ computed for the short-range Yukawa potential $\mathrm{V}(\mathrm{x})=-\mathrm{V}_{0} \exp (-|\mathrm{x}|)$.

as a function of the box size L. It seems clear that these coefficients indeed become independent of $\mathrm{L}$ as it increases. As a side remark, we note that (with the exception of the $\alpha_{5}$ that also seems to decrease with $\mathrm{L}$ ) the magnitude of the coefficients decrease with the order rather rapidly for a given $\mathrm{L}$. The dips for specific values of $L$ in $\alpha_{4}, \alpha_{6}$ and $\alpha_{7}$ are associated with our logarithmic axis therefore reflect regions where the coefficients change their sign. To stress the universality of the rather L-independent behavior of these effective coefficients $\alpha_{\mathrm{n}}$, we have examined them also for a finite range potential $\mathrm{V}(\mathrm{x})=\mathrm{V}_{0}$ $[\operatorname{Sign}(x-a)-\operatorname{Sign}(x+a)] / 2$, where $\operatorname{Sign}(y)$ denotes the function $x /|x|$. While here the coefficients are even more oscillatory for small $\mathrm{L}$, the $\alpha_{\mathrm{n}}$ also approach L-independent values for sufficiently large L.

The most interesting case occurs for the zero-range potential $\mathrm{V}(\mathrm{x})=-\mathrm{v}_{0} \delta(\mathrm{x})$. In this case we find numerically that all coefficients $\alpha_{n}$ are entirely independent of $\mathrm{L}$. As we briefly summarize in the next section, in this case it is actually possible to use the first M numerical coefficients $\alpha_{\mathrm{n}}$ for a truncated Borel summation technique to recover the exact binding energy.

\subsection{Diverging perturbative expansion for the bound state energy}

As a specific example, we will use the diverging perturbative expansion for the ground state of the 
zero-range potential $\mathrm{V}(\mathrm{x})=-\mathrm{v}_{0} \delta(\mathrm{x})$ to predict the ground state energy, which in the limit of $\mathrm{L} \rightarrow \infty$ approaches $\mathrm{E}_{\mathrm{g}}\left(\mathrm{v}_{0}\right)=-\mathrm{v}_{0}^{2} / 2$.

The usual Borel summation technique [7,8] is defined by interchanging the integration over an auxiliary variable $t$ and the infinite summation over $n$ in the equality $\Sigma_{n=0}^{\infty} b_{n}=\Sigma_{n=0}^{\infty} b_{n} / n ! n !=\Sigma_{n=0}^{\infty} b_{n} / n ! \int_{0}^{\infty} d t$ $\exp (-t) t^{n}=\int_{0}^{\infty} d t \exp (-t) B(t)$. We therefore have the Borel transformed sum given as $B(t) \equiv \sum_{n}=0 b_{n} / n !$ $\mathrm{t}^{\mathrm{n}}$. This general summation method $[3,11,12]$ can work well, if e.g., we are able to find analytical expressions for the $n$-dependence of all the coefficients $b_{n}$.

From a practical point the more interesting question is whether it is also possible to estimate the finite value of the diverging sum, if one does not know analytically how each perturbative expansion coefficient $b_{n}$ depends on the order $\mathrm{n}$. In many cases, these coefficients can only be determined numerically and therefore only the first $M$ coefficients can be computed leading to $B_{M}(t) \equiv \Sigma_{n=0}{ }^{M} b_{n} / n$ ! $t^{n}$. As for any finite $\mathrm{M}$ the full inverse Borel summation technique reproduces exactly the partial sum of the perturbative expansion, it therefore would predict precisely the (diverging!) result of the M-th partial sum. So it would return the rather large value $\int_{0}^{\infty} d t \exp (-t) B_{M}(t)=\Sigma_{n=0}{ }^{M} b_{n}$, associated with the M-th (diverging) partial sum and not the true finite value for $\mathrm{M} \rightarrow \infty$.

In the following we will propose an approximate numerical approach that will permit us to estimate the true finite limit of a diverging perturbative expansion based on only the first $\mathrm{M}$ coefficients. Our proposed technique is based on the observation that due to the exponential factor exp(-t) the integrand in the inverse Borel (Laplace) transformation decreases rapidly with $t$, such that in many cases only smaller values of the integration variable $\mathrm{t}$ from $\mathrm{B}(\mathrm{t})$ provide the main contribution. While the truncated Borel transformation, defined as $\mathrm{B}_{\mathrm{M}}(\mathrm{t}) \equiv \Sigma_{\mathrm{n}=1}{ }^{\mathrm{M}} \mathrm{a}_{\mathrm{n}} / \mathrm{n}$ ! $\mathrm{t}^{\mathrm{n}}$, usually grows with increasing $\mathrm{t}$, due to the factorial in the denominator this sum can be converging for a given parameter $t$. If it is converging, the proposed method below can work very well. If the upper limit of the inverse Borel transform is truncated to a suitably chosen finite limit, defined as $\mathrm{Z}$, we can approach the original finite value associated with the original infinite summation $\int_{0} \mathrm{Z} d \mathrm{t}$ $\exp (-t) B_{M}(t) \approx \sum_{n=0}^{\infty} b_{n}$. It turns out that the finite limit of this diverging sum $\Sigma_{n=0}^{\infty} b_{n}$ is contained already in the integrand (and its integral) $\exp (-\mathrm{t}) \mathrm{B}_{\mathrm{M}}(\mathrm{t})$ for rather small values of $\mathrm{t}$. The resulting large (and unphysical) value of any finite partial sum $\Sigma_{n=0} M b_{n}$ manifests itself only for large values of $t$. It is therefore 
possible to simply restrict the original integration domain $\int_{0}^{\infty} \mathrm{dt}$ to $\int_{0}^{\mathrm{Z}} \mathrm{dt}$ to avoid the large value associated with a finite (and not infinite) M. We will provide the relevant rigorous mathematical proofs, the limitation and the general applicability of this algorithm in a separate work and just present here a concrete example as a first proof of concept.

We have computed numerically the first $M=50$ Rayleigh-Schrödinger perturbative terms for the partial sum $\Sigma_{\mathrm{n}=0} \mathrm{M}_{\mathrm{n}}=\Sigma_{\mathrm{n}=0}{ }^{\mathrm{M}} \mathrm{e}_{\mathrm{n}} \mathrm{v}_{0}{ }^{\mathrm{n}}$ for the expansion of the potential $\mathrm{V}(\mathrm{x})=-\mathrm{v} 0 \delta(\mathrm{x})$ with $\mathrm{v}_{0}=4$ in a numerical box of length $\mathrm{L}=2$ a.u. As the sum rapidly diverges, the 50-th partial sum amounts to $\Sigma_{\mathrm{n}=0} 50 \mathrm{~b}_{\mathrm{n}}=8.23 \times 10^{6}$. We have then computed the truncated Borel sum $B_{50}(t) \equiv \Sigma_{n=0}^{50} b_{n} / n ! t^{n}$ as a function of $t$. The integrand $\exp (-\mathrm{t}) \mathrm{B}_{50}(\mathrm{t})$ decreases from zero $($ at $\mathrm{t}=0)$ to about -2 for $\mathrm{t} \approx 2.2$ and then approaches zero at around $\mathrm{t}=10$, where it stays until $t \approx 20$, after which it grows significantly to maximum of about $5 \times 10^{5}$ (at $t \approx 50$ ) and then decreases back to zero. As an arbitrary measure of where to truncate the integration region we have chosen here that value for $t$, where the integrand changes its sign from negative to positive. This root, defined as $\exp (-Z) B_{50}(Z)=0$ led to $Z=10.9228766$. The truncated inverse Borel transformation, $\int_{0} Z d t \exp (-t) B_{50}(t)$ was computed to be -7.99 , which differs from the exact analytical bound state energy $E_{g}\left(v_{0}=4\right)=-4^{2} / 2$ by less than $0.12 \%$. This illustrates that it is indeed possible to approximate the ground state energy from the partial sums of the diverging perturbative expansion. It also demonstrates that while each perturbative term depends on $\mathrm{L}$, the finite value of the diverging series does not.

\section{Avoided level crossings: Converging and diverging perturbative regimes}

Here we will examine the validity of perturbation theory for a pair of energy eigenvalues close to an avoided crossing [4-6]. We focus on two cases of avoided crossings, in the first scenario the perturbation will cause two unperturbed levels to repel each other, while in the second scenario the two levels first approach each other, before they repel. As the major contributions to the unperturbed energies are due to the coupling with states in the immediate energy vicinity, we restrict the Hilbert space to those two states. For simplicity and without any loss of generality, we assume that the unperturbed energies are $\mathrm{E}_{2}\left(\mathrm{~V}_{0}=0\right)=\mathrm{E}_{2}$ and $\mathrm{E}_{1}\left(\mathrm{~V}_{0}=0\right)=0$ and correspond to the unperturbed eigenstates $(1,0)$ and $(0,1)$.

\subsection{Perturbation theory at the avoided crossing, analytical and numerical Borel summation}

In the first scenario we assume that coupling potential matrix is given by $\mathrm{V}=\mathrm{V}_{0}((0, \kappa),(\kappa, 0))$, with a dimensionless correlation strength $\kappa$, such that the corresponding total Hamiltonian $2 \times 2$ matrix is given by 
$H\left(V_{0}\right)=\left(\left(E_{2}, V_{0} \kappa\right),\left(V_{0} \kappa, 0\right)\right)$. As the Hamiltonian matrix is only $2 \times 2$, we can obtain the analytical form of the lower energy branch as $\mathrm{E}_{1}\left(\mathrm{~V}_{0}\right)=\mathrm{E}_{2} / 2-\left[\mathrm{E}_{2}^{2}+4 \kappa^{2} \mathrm{~V}_{0}^{2}\right]^{1 / 2} / 2$, which with $\mathrm{y} \equiv\left(2 \kappa \mathrm{V}_{0} / \mathrm{E}_{2}\right)^{2}$ can be trivially rescaled as $E_{1}\left(V_{0}\right)=E_{2} / 2\left[1-(1+y)^{1 / 2}\right]$. A perturbative approach based on the Rayleigh-Schrödinger perturbation theory (as reviewed in Appendix B) in the parameter y would lead to the following general expansion for this energy

$$
\mathrm{E}_{1}\left(\mathrm{~V}_{0}\right)=\mathrm{E}_{2} / 2 \quad \Sigma_{\mathrm{n}=1}^{\infty} \mathrm{e}_{\mathrm{n}} \mathrm{y}^{\mathrm{n}}
$$

In this particular model we know that the coefficients $e_{n}$ are the binomial coefficient $a_{n}=B(1 / 2, n)$, defined as $\mathrm{B}(\tau, \mathrm{n})=\tau(\tau-1)(\tau-2) . .(\tau-\mathrm{n}+1) / \mathrm{n}$ !. Using the conventional quotient criterion to estimate the radius of convergence, we would predict that for $|\mathrm{y}|>\lim _{\mathrm{n} \rightarrow \infty} \mathrm{B}(1 / 2, n) / \mathrm{B}(1 / 2, \mathrm{n}+1)$ the power series should become divergent, in other words, we can no longer estimate the finite value of the series by using the partial sum technique. As the ratio is simply $2(1+n) /(1-2 n)$, the radius of convergence amounts to $|y|=1$, or equivalently, $\mathrm{V}_{0}=\mathrm{E}_{2} /(2 \kappa)$, which is proportional to the ratio of the energy difference of the two unperturbed energies to the coupling strength. Therefore, for $\mathrm{V}_{0}>\mathrm{E}_{2} /(2 \kappa)$ the series becomes divergent. However, it turns out that even for $\mathrm{V}_{0}>\mathrm{E}_{2} /(2 \kappa)$ the diverging sum can be summed up leading to a finite value. For instance, this can be done by using the Borel summation technique described in Sec. 2.2. In order to calculate this sum numerically, it is generally necessary to determine the functional dependence of the $n$-th perturbative expansion coefficients $e_{n}$ for all $n$. In our case, we know these exactly and therefore can obtain an explicit form of the Borel transformation as

$$
\mathrm{B}(\mathrm{yt})=\Sigma_{\mathrm{n}=1}^{\infty} \mathrm{e}_{\mathrm{n}} / \mathrm{n} !(\mathrm{t} \mathrm{y})^{\mathrm{n}}=-1+\exp (-\mathrm{yt} / 2)\left[\left(1+\mathrm{yt} \mathrm{I}_{0}(\mathrm{yt} / 2)+\mathrm{yt} \mathrm{I}_{1}(\mathrm{yt} / 2)\right]\right.
$$

where $\mathrm{I}_{0}()$ and $\mathrm{I}_{1}()$ denote the modified Bessel functions of the first kind of orders zero and one. When we apply the Laplace transform (the inverse Borel transformation), we obtain

$$
\begin{aligned}
\int_{0}^{\infty} \mathrm{dt} \exp (-\mathrm{t}) \mathrm{B}(\mathrm{yt}) & =\int_{0}^{\infty} \mathrm{dt} \exp (-\mathrm{t})\left\{-1+\exp (-\mathrm{yt} / 2)\left[\left(1+\mathrm{yt} \mathrm{I}_{0}(\mathrm{yt} / 2)+\mathrm{yt} \mathrm{I}_{1}(\mathrm{yt} / 2)\right]\right\}\right. \\
& =1-(1+\mathrm{y})^{1 / 2}
\end{aligned}
$$


We can therefore use the perturbative data to predict the correct energy eigenvalue for any perturbation strength $\mathrm{V}_{0}$, even in a domain where the series is formally diverging.

We once again return to the more interesting case, where only a limited number of partial sums are accessible. Here we test again the truncated Borel summation algorithm as outlined in Section 2.2. To provide a simple example, the divergent series $\mathrm{E}_{1}=\sum_{\mathrm{n}=1}^{\infty} \mathrm{a}_{\mathrm{n}} \mathrm{y}^{\mathrm{n}}$ where the coefficients $\mathrm{a}_{\mathrm{n}}$ are the binomial coefficients $\mathrm{B}(1 / 2, \mathrm{n})$ is identical to $(1+\mathrm{y})^{1 / 2}$. For example, the value for $\mathrm{y}=3$ (which is three times larger than the radius of convergence 1 ) the exact value of the diverging sum is obviously $E_{1}=2$ a.u.. If we use only the first $M=20$ expansion coefficients terms (with root $Z=4.16410838$ ) we obtain numerically $E_{1}=$ 1.91524589 a.u., corresponding to a relative error of $4.2 \%$ compared to the exact value $E_{1}=2$ a.u.. We note that the corresponding 20-th partial sum $\Sigma_{n=1}^{20} a_{n} 3^{n}$ would amount to $-8.2 \times 10^{6}$ a.u. The accuracy of the truncated Borel technique is even more impressive, if we take the first $M=100$ expansion coefficients terms (with root $Z=14.0675803$ ) into consideration. Here we obtain $E_{1}=1.99999227$ a.u., corresponding to a relative error of less than $0.0004 \%$.

\subsection{Perturbation theory towards the avoided crossing: numerical Borel summation}

In the second scenario we have assumed that the coupling potential matrix is given by $\mathrm{V}=\mathrm{V}_{0}((-1$, $\kappa),(\kappa, 0))$, such that the corresponding total Hamiltonian $2 \times 2$ matrix is given by $H\left(V_{0}\right)=\left(\left(1-V_{0}, V_{0} \kappa\right),\left(V_{0} \kappa\right.\right.$, $0)$ ). In the trivial special case of no correlation between the states $(\kappa=0)$, the perturbed energies are $\mathrm{E}_{1}\left(\mathrm{~V}_{0}\right)=0$ and $\mathrm{E}_{2}\left(\mathrm{~V}_{0}\right)=1-\mathrm{V}_{0}$, such that the two eigenvalues would cross (become momentarily degenerate) at $\mathrm{V}_{0}=1$ while the corresponding two eigenstates maintain their form $(1,0)$ and $(0,1)$.

If we now assume that $\kappa$ is non-zero but very small, the behavior of the eigenstates remains qualitatively the same, except that for $\mathrm{V}_{0} \approx 1$ the two energies do not cross and therefore reveal an avoided crossing. More quantitatively, the two levels have their smallest spacing of $2 \kappa /\left(1+4 \kappa^{2}\right)^{1 / 2}$ at $V_{0}=1 /\left(1+4 \kappa^{2}\right)$. As a result, the lower level is effectively pushed to lower energies as if it was repelled by the upper intruder state, which in turn is pushed to higher energies. It is important to note that due to the avoided crossing the states associated with each energy branch change their character entirely. For $\kappa<<1$ the lower energetic eigenstate $(0,1)$ changes adiabatically to $(1,0)$ after the avoided crossing. The question is therefore valid if perturbation 
theory is at all able to describe this transition from $(0,1)$ to $(1,0)$ as $\mathrm{V}_{0}$ is increased. We note that exactly at the avoided crossing $\left[\mathrm{V}_{0}=\left(1+4 \kappa^{2}\right)^{-1}\right]$ the eigenstate takes the form $(1,-1)$, which shows the large amount of state mixing. Figure 5 summarizes this behavior.

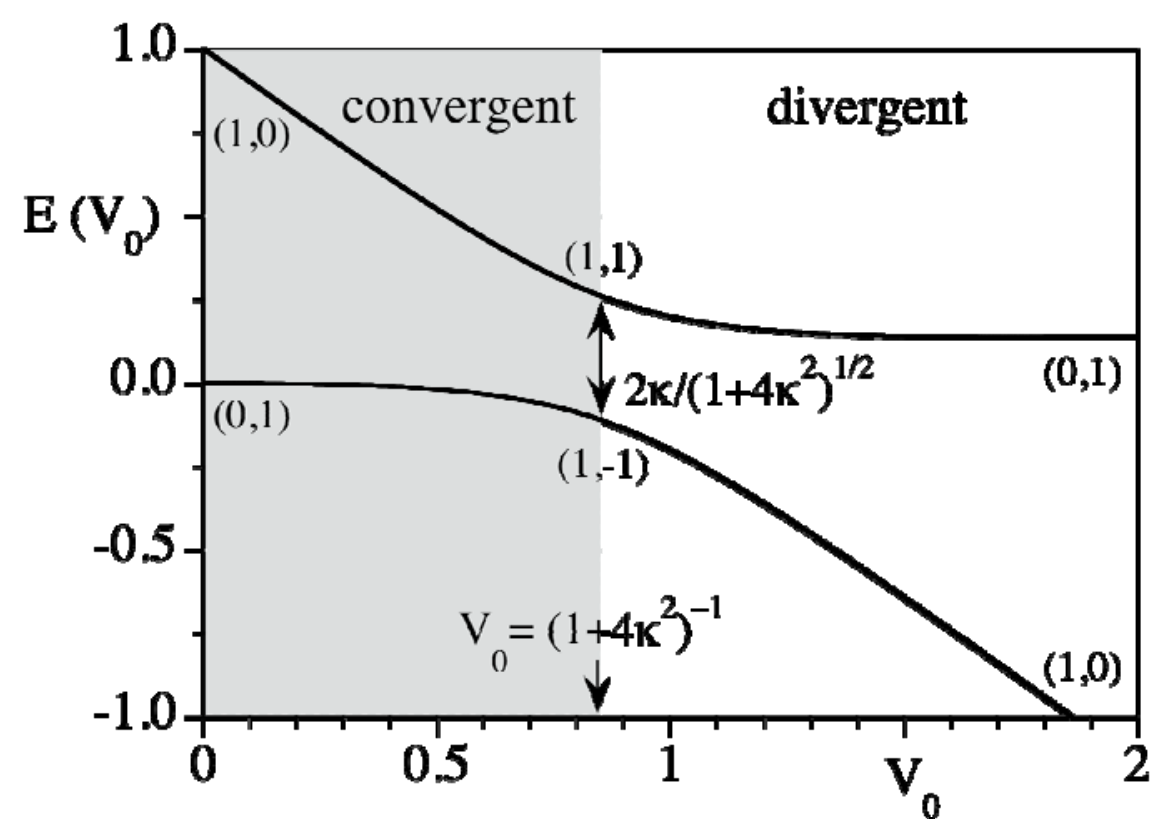

Figure 5 The avoided crossing for two neighboring energy levels. We also show the corresponding eigenstates in the limit of small $\kappa$. (Energies are shown for $\kappa=0.2$ a.u.)

As the Hamiltonian matrix is only $2 \times 2$, we can obtain again the analytical form of the lower energy branch as

$$
\mathrm{E}_{1}\left(\mathrm{~V}_{0}\right)=\left(1-\mathrm{V}_{0}\right) / 2-\left[\left(1-\mathrm{V}_{0}\right)^{2}+4 \kappa^{2} \mathrm{~V}_{0}^{2}\right]^{1 / 2} / 2
$$

There are two ways in which this problem could be handled perturbatively, the first one would assume that the states and energies at the avoided crossing are precisely known. Here the square root in Eq. (3.4) can be rewritten in the simplified form $\left[\left(1-\mathrm{V}_{0}\right)^{2}+4 \mathrm{\kappa}^{2} \mathrm{~V}_{0}^{2}\right]^{1 / 2} / 2=\xi\left[1+\mathrm{y}^{2}\right]^{1 / 2}$, where we have introduced a new rescaled and shifted perturbation parameter $\mathrm{y} \equiv\left(4 \kappa^{2}+1\right) /(2 \kappa) \mathrm{V}_{0}-1 /(2 \kappa)$ and $\xi \equiv \kappa /\left(1+4 \kappa^{2}\right)^{1 / 2}$, which is precisely half the minimum energy distance at the avoided crossing. As we have seen above, for $V_{\min }$ $\mathrm{d}=1 /\left(1+4 \kappa^{2}\right)$ we have the minimum distance corresponding to $\mathrm{y}=0$. A perturbative approach based on the Rayleigh-Schrödinger perturbation theory [2] (as reviewed in Appendix B) in the parameter y would lead to the following expansion for this energy 


$$
\mathrm{E}_{1}\left(\mathrm{~V}_{0}\right)=\left(1-\mathrm{V}_{0}\right) / 2-\xi \Sigma_{\mathrm{n}=0}^{\infty} \mathrm{B}(1 / 2, \mathrm{n}) \mathrm{y}^{\mathrm{n}}
$$

where $\mathrm{B}(1 / 2, \mathrm{n})$ denotes again the binomial coefficient. This situation then is directly related to the one discussed in the Section 3.1 above. Here the radius of convergence amounts to $|y|=\mid\left(4 \kappa^{2}+1\right) /(2 \kappa) V_{0}-$ $1 /(2 \kappa) \mid=1$ leading to the range $\mathrm{V}_{0}=\mathrm{V}_{\min \mathrm{d}} \pm 2 \kappa /\left(4 \kappa^{2}+1\right)$.

The conceptually more interesting case would be to view the original states and energies for $\mathrm{V}_{0}=0$ as unperturbed. In this case it is presently not clear, how one could obtain a closed form analytical expression for the corresponding perturbative expansion coefficients. However, numerically, they can certainly be obtained as the Taylor expansion coefficients $\mathrm{e}_{\mathrm{n}}=\mathrm{d}^{\mathrm{n}} \mathrm{E}_{1}\left(\mathrm{~V}_{0}\right) / \mathrm{dV}_{0}{ }^{\mathrm{n}} \mid \mathrm{v} 0=0$

$$
\mathrm{E}_{1}\left(\mathrm{~V}_{0}\right)=\Sigma_{\mathrm{n}=0}^{\infty} \quad \mathrm{e}_{\mathrm{n}} \mathrm{V}_{0}{ }^{\mathrm{n}}=\Sigma_{\mathrm{n}=0}^{\infty} \quad \mathrm{d}^{\mathrm{n}} \mathrm{E}_{1}\left(\mathrm{~V}_{0}\right) / \mathrm{dV}_{0}{ }^{\mathrm{n}} \mid \mathrm{V}_{0}=0 \quad \mathrm{~V}_{0}{ }^{\mathrm{n}}
$$

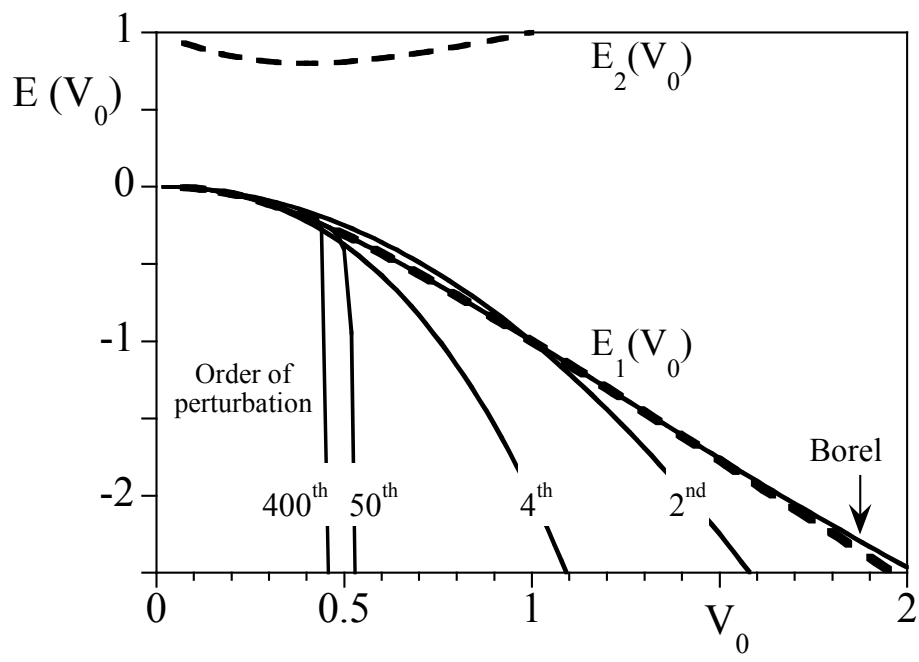

Figure 6 The perturbative partial sums up to four different orders $M$ to predict the lower energy at the avoided level crossing. For comparison and reference, the dashed lines are the exact two energies. We also show the predictions of the numerical truncated Borel summation technique. $(\kappa=1)$

In order to show the applicability of ordinary perturbation theory, we have displayed the prediction of the $\mathrm{M}=2^{\text {nd }}, 4^{\text {th }}, 50^{\text {th }}$ and $400^{\text {th }}$ order perturbative expansions. While the series certainly matches the exact energies very well for small $\mathrm{V}_{0}$, the highest order series shows that for $\mathrm{V}_{0}>0.45$, the series diverges. It is quite remarkable and maybe even unexpected that even a polynomial of order 400 corresponding to 401 free adjustable coefficients is not able to match this rather nicely behaved dashed line for a larger range of $\mathrm{V}_{0}$. 
We have also included in the figure the predictions of the numerical truncated Borel summation technique based on $M=40$ terms that we introduced in the Section 3.1. It clearly shows that it works rather well to estimate the energies far beyond the parameter regime for which the perturbative series is convergent. However, in order to follow the entire energy path, higher and higher perturbative expansions are required.

\section{Summary and outlook}

In this work we have shown that perturbation theory is fully applicable to predict the occurrence of bound states for the weakly coupling region where the spatial extent of the bound state wave function is comparable to the size of the numerical box. As the size of this box L is increased, the radius of convergence of the perturbative expansion decreases and the perturbative expansion becomes naturally divergent. In this diverging regime we proposed a generalized truncated Borel summation technique to compute the finite energy of the ground state. We will devote a future publication to examine the range of applicability of this technique based on a finite number of partial sums for various finite-range, short-range and also possibly long-range potentials. We will also examine how it compares to other techniques, for which the Borel transformed expression is estimated by Pade-approximations of various orders $[11,12]$. We have shown that the zero-range potential is an excellent candidate for this method and reiterate that for this particular potential all perturbative expansion coefficients have a simple scaling $\mathrm{e}_{\mathrm{n}} \sim \mathrm{L}^{\mathrm{n}-2}$ with the box size $\mathrm{L}$.

The finite box size $\mathrm{L}$ acts as a regularization parameter. We should note that while we have presented our results for a simple systems in one-spatial dimension, the specific scaling of the perturbative expansion coefficients $\mathrm{e}_{\mathrm{n}}$ with $\mathrm{L}$ would need to be generalized to higher dimensions. This regularization also is directly related to renormalization techniques for example in quantum field theoretical lattice calculations, where the momentum grid spacing is inversely proportional to $\mathrm{L}$.

We should point out that a rigorous treatment of bound states is still a difficult subject [13]. Bound states are believed not to exist perturbatively as such problems necessarily involve a breakdown of ordinary perturbation theory. Therefore the associated poles of the $\mathrm{S}$ matrix can only arise from a divergence of the sum of infinitely many Feynman diagrams [14]. We also note that the Bethe-Salpeter equation to approximate bound state energies from a quantum field theory is obtained from lowest order perturbation theory [15]. While some early work has provided existence proofs that for certain quantum field theoretical systems bound state energies can be obtained by Borel summation techniques [16], the actual determination of these energies from a divergent Feynman perturbation theory remains difficult.

For the simpler system of an avoided crossing where all expansion coefficients are known analytically, 
the powerful Borel summation technique can be applied to reproduce exactly the eigenvalues from the diverging sum. For more generic situations where there are no closed form solution for the expansion coefficients and the precise $n$-dependence of the $n$-th order expansion terms can only be provided consecutively on a term by term basis numerically, the truncated Borel technique can be applied successfully. It works well when the corresponding Borel transform converges, but is an interesting challenge to examine this technique and its possible generalizations for other (not-exponentially based) moment-based summation techniques.

\section{Acknowledgements}

We would like to thank C. Bender for several helpful discussions and pointing out Ref. 2. This work has been supported by the NSF and the NSFC (\#11529402). 


\section{Appendix A Numerical diagonalization}

In order to convert the Hamiltonian to a finite matrix, we have confined the particle spatially to a numerical box from $-\mathrm{L} / 2$ to $\mathrm{L} / 2$ in which we discretize the spatial coordinate into $\mathrm{D}$ points, corresponding to a grid spacing of $\Delta x=L / D$. As a result, the momenta are discretized with a spacing $\Delta k=2 \pi / L$. In the spatial representation, we have approximated the Hamiltonian [9]

$$
\left\langle\mathrm{x}_{\alpha}\left|\mathrm{p}^{2} / 2+\mathrm{V}(\mathrm{x})\right| \mathrm{x} \beta\right\rangle=\mathrm{D}^{-1} \sum_{\mathrm{n}=1} \mathrm{D} \mathrm{k}_{\mathrm{n}}^{2} / 2 \exp \left[\mathrm{i} \mathrm{k}_{\mathrm{n}}\left(\mathrm{x} \beta-\mathrm{x}_{\alpha}\right)\right]+\mathrm{x}_{\alpha} \mid \mathrm{p}^{2} / 2+\mathrm{V}\left(\mathrm{x}_{\alpha}\right) \delta_{\alpha, \beta}
$$

This representation was used to calculate the spatial probabilities of the energy eigenstates. With regard to the perturbative results, it is more advantageous to express the Hamiltonian in its momentum representation. This is equivalent to the unperturbed energy eigenbasis for which the kinetic part of the Hamiltonian is diagonal, whereas the Fourier transformed potential $\mathrm{W}(\mathrm{k}) \equiv \mathrm{L}^{-1} \int \mathrm{dk} \exp (-\mathrm{i} \mathrm{k} x) \mathrm{V}(\mathrm{x})$ couples basically all states.

$$
\left\langle\mathrm{k}_{\alpha}\left|\mathrm{p}^{2} / 2+\mathrm{V}(\mathrm{x})\right| \mathrm{k} \beta\right\rangle=\mathrm{k}_{\alpha}^{2} / 2 \delta \alpha, \beta+\mathrm{W}\left(\mathrm{k}_{\alpha}-\mathrm{k} \beta\right)
$$

In this work, we have examined three attractive potentials, the short-range Yukawa potential $\mathrm{V}_{\mathrm{Y}}(\mathrm{x})=-\mathrm{V}_{0}$ $\exp (-\mathrm{b}|\mathrm{x}|)$, the finite-range potential $\mathrm{V}_{\mathrm{F}}(\mathrm{x})=\mathrm{V}_{0}[\operatorname{Sign}(\mathrm{x}-\mathrm{a})-\operatorname{Sign}(\mathrm{x}+\mathrm{a})] / 2$, where $\operatorname{Sign}(\mathrm{y})$ denotes the function $\mathrm{x} /|\mathrm{x}|$ and also the zero-range potential $\mathrm{VD}(\mathrm{x})=-\mathrm{v}_{0} \delta(\mathrm{x})$. We have then Fourier transformed these potentials and evaluated them at the numerical momentum grid points, leading to

$$
\begin{aligned}
& \mathrm{W}_{\mathrm{Y}}\left(\mathrm{k}_{\alpha}-\mathrm{k} \beta\right)=-(2 / \mathrm{L}) \mathrm{V}_{0} \mathrm{~b}^{2} /\left[\left(\mathrm{k}_{\alpha}-\mathrm{k} \beta\right)^{2}+\mathrm{b}^{2}\right] \\
& \mathrm{W}_{\mathrm{F}}\left(\mathrm{k}_{\alpha}-\mathrm{k} \beta\right)=-(2 / \mathrm{L}) \mathrm{V}_{0} \operatorname{Sin}\left[\mathrm{a}\left(\mathrm{k}_{\alpha}-\mathrm{k} \beta\right)\right] /\left(\mathrm{k}_{\alpha}-\mathrm{k} \beta\right) \\
& \mathrm{W}_{\mathrm{D}}\left(\mathrm{k}_{\alpha}-\mathrm{k} \beta\right)=-(1 / \mathrm{L}) \mathrm{v}_{0}
\end{aligned}
$$

While most of the eigenvalues associated with the quasi-continuum portion of the spectrum depend on L, we have chosen the matrix dimension D large enough, such that the relevant eigenvalues are converged. 


\section{Appendix B Raleigh-Schrödinger perturbation theory}

As the Raleigh-Schrödinger perturbation theory is well-known [1-3], we just briefly summarize here its derivation and use this appendix mainly to introduce our notation. In order to find a perturbative expression for the lowest order eigenvalue $\left(\mathrm{H}_{0}+\lambda \mathrm{V}\right)|\Omega(\lambda)\rangle=\Omega(\lambda)|\Omega(\lambda)\rangle$, we use the usual Ansatz for the eigenstate $|\Omega(\lambda)\rangle$ and eigenvalue $\Omega(\lambda)$

$$
\begin{aligned}
& |\Omega(\lambda)\rangle=\left|\omega^{(0)}\right\rangle+\lambda\left|\omega^{(1)}\right\rangle+\lambda^{2}\left|\omega^{(2)}\right\rangle+\ldots \\
& \Omega(\lambda)=\omega^{(0)}+\lambda \omega^{(1)}+\lambda^{2} \omega^{(2)}+\ldots
\end{aligned}
$$

In order to quickly address the issue of normalization, we arbitrarily assume that $\langle\Omega \mid \Omega\rangle=1$ and $\left\langle\omega^{(0)} \mid \omega^{(0)}\right\rangle$ $=1$. Up to first order in $\lambda$, this leads to $1=\left(\left\langle\omega^{(0)}\left|+\lambda\left\langle\omega^{(1)}\right|\right)\left(\left|\omega^{(0)}\right\rangle+\lambda\left|\omega^{(1)}\right\rangle\right)\right.\right.$. Multiplying this out, dropping the second-order term $\lambda^{2}\left\langle\omega^{(1)} \mid \omega^{(1)}\right\rangle$, and using $\left\langle\omega^{(0)} \mid \omega^{(0)}\right\rangle=1$ this corresponds to $\left\langle\omega^{(0)} \mid \omega^{(1)}\right\rangle+$ $\left\langle\omega^{(1)} \mid \omega^{(0)}\right\rangle=0$. If we assume that $\mathrm{H}$ is Hermitian, we can choose the states real and obtain $\left\langle\omega^{(0)} \mid \omega^{(1)}\right\rangle=0$. We also arbitrarily fix the normalization of $|\Omega\rangle$ to fulfill $\left\langle\omega^{(0)} \mid \Omega\right\rangle=1=>\left\langle\omega^{(0)} \mid \omega^{(\mathrm{n})}\right\rangle=0$ for all $\mathrm{n}$, except $\mathrm{n}=0$.

We can insert these expressions (B1) into the eigenvalue equation and equating equal powers of the expansion parameter $\lambda$. Comparing term by term we obtain:

$$
\begin{aligned}
& \left(\mathrm{H}_{0}-\omega^{(0)}\right)\left|\omega^{(0)}\right\rangle=0 \\
& \left(\mathrm{H}_{0}-\omega^{(0)}\right)\left|\omega^{(1)}\right\rangle+\left(\mathrm{V}-\omega^{(1)}\right)\left|\omega^{(0)}\right\rangle=0 \\
& \left(\mathrm{H}_{0}-\omega^{(0)}\right)\left|\omega^{(2)}\right\rangle+\left(\mathrm{V}-\omega^{(1)}\right)\left|\omega^{(1)}\right\rangle-\omega^{(2)}\left|\omega^{(0)}\right\rangle=0 \\
& \left(\mathrm{H}_{0}-\omega^{(0)}\right)\left|\omega^{(\mathrm{n})}\right\rangle+\left(\mathrm{V}-\omega^{(1)}\right)\left|\omega^{(\mathrm{n}-1)}\right\rangle-\omega^{(2)}\left|\omega^{(\mathrm{n}-2)}\right\rangle-\omega^{(3)}\left|\omega^{(\mathrm{n}-3)}\right\rangle \ldots-\omega^{(\mathrm{n})}\left|\omega^{(0)}\right\rangle=0
\end{aligned}
$$

If we multiply the general Eq. (B2d) with $\left\langle\omega^{(0)}\right|$ from the left and use $\left\langle\omega^{(0)} \mid \omega^{(n)}\right\rangle=0$ for any n $\neq 0$, we can solve for $\omega^{(\mathrm{n})}$ and obtain for the eigenvalue correction 


$$
\omega^{(\mathrm{n})}=\left\langle\omega^{(0)}|\mathrm{V}| \omega^{(\mathrm{n}-1)}\right\rangle
$$

Next we need to find $\left|\omega^{(\mathrm{n})}\right\rangle$ from Eq. (B2d). It is of the form $\left(\omega^{(0)}-\mathrm{H}_{0}\right)\left|\omega^{(\mathrm{n})}\right\rangle \equiv|\mathrm{RHS}\rangle$ where we have defined the (known) right-hand side state as $|\mathrm{RHS}\rangle=\left(\mathrm{V}-\omega^{(1)}\right)\left|\omega^{(\mathrm{n}-1)}\right\rangle-\omega^{(2)}\left|\omega^{(\mathrm{n}-2)}\right\rangle-\omega^{(3)}\left|\omega^{(\mathrm{n}-3)}\right\rangle \ldots$ $-\omega^{(\mathrm{n})}\left|\omega^{(0)}\right\rangle$. This equation needs to be solved (inverted) for $\left|\omega^{(\mathrm{n})}\right\rangle$. We do this by first (scalar) multiplying both sides with $\left\langle\beta^{(0)}\right|$ leading to $\left(\omega^{(0)}-\beta^{(0)}\right)\left\langle\beta^{(0)} \mid \omega^{(\mathrm{n})}\right\rangle=\left\langle\beta^{(0)} \mid \mathrm{RHS}\right\rangle$. After dividing both sides by the non-zero factor $\left(\omega^{(0)}-\beta^{(0)}\right)$ we (ordinary) multiplying both sides with the state $\left|\beta^{(0)}\right\rangle$ leading to $\left|\beta^{(0)}\right\rangle\left\langle\beta^{(0)} \mid \omega^{(\mathrm{n})}\right\rangle=\left|\beta^{(0)}\right\rangle\left(\omega^{(0)}-\beta^{(0)}\right)^{-1}\left\langle\beta^{(0)} \mid \mathrm{RHS}\right\rangle$. We then sum both sides over all $\beta^{(0)}$, where the singular term $\beta^{(0)}=\omega^{(0)}$ is omitted from the sum. Using the relationship $\Sigma_{\beta}\left|\beta^{(0)}\right\rangle\left\langle\beta^{(0)}|=1-| \omega^{(0)}\right\rangle\left\langle\omega^{(0)}\right|$ we obtain on the left hand side $\sum_{\beta}\left|\beta^{(0)}\right\rangle\left\langle\beta^{(0)} \mid \omega^{(\mathrm{n})}\right\rangle=\left(1-\left|\omega^{(0)}\right\rangle\left\langle\omega^{(0)}\right|\right)\left|\omega^{(\mathrm{n})}\right\rangle=\left|\omega^{(\mathrm{n})}\right\rangle$, while the right hand side results in $\left|\omega^{(n)}\right\rangle=\Sigma_{\beta}\left|\beta^{(0)}\right\rangle\left(\omega^{(0)}-\beta^{(0)}\right)^{-1}\left\langle\beta^{(0)} \mid \mathrm{RHS}\right\rangle$. To simplify our notation we introduce a "truncated resolvent", defined as $\mathrm{G} \equiv \Sigma_{\beta}\left|\beta^{(0)}\right\rangle\left(\omega^{(0)}-\beta^{(0)}\right)^{-1}\left\langle\beta^{(0)}\right|$, which differs from the usual resolvent $\mathrm{G}(\mathrm{H}, \mathrm{z})=1 /(\mathrm{z}-\mathrm{H})$ in two ways. First, it is evaluated at precisely $\mathrm{z}$ equal to an eigenvalue of $\mathrm{H}$. Second the action of the specific eigenstate $\left|\omega^{(0)}\right\rangle$ on $\mathrm{G}$ vanishes. So it is a true resolvent only with respect to the orthogonal complementary space that excludes the state $\left|\omega^{(0)}\right\rangle$. So our final result is $\left|\omega^{(\mathrm{n})}\right\rangle=\mathrm{G}|\mathrm{RHS}\rangle$. The last term on the right-hand side vanishes as $\mathrm{G}\left|\omega^{(0)}\right\rangle=0$.

$$
\left|\omega^{(\mathrm{n})}\right\rangle=\mathrm{G}\left(\mathrm{V}-\omega^{(1)}\right)\left|\omega^{(\mathrm{n}-1)}\right\rangle-\omega^{(2)} \mathrm{G}\left|\omega^{(\mathrm{n}-2)}\right\rangle-\omega^{(3)} \mathrm{G}\left|\omega^{(\mathrm{n}-3)}\right\rangle \ldots-\omega^{(\mathrm{n}-1)} \mathrm{G}\left|\omega^{(1)}\right\rangle(\mathrm{B} 4
$$

Once the two finite dimensional matrices $\mathrm{G}$ and $\mathrm{V}$ together with the vector $\left|\omega^{(0)}\right\rangle$ are computed in the energy basis of the unperturbed states, one can iterate Eqs. (B3) and (B4) to obtain numerically the perturbative energy corrections $\omega^{(\mathrm{n})}$ for practically arbitrary orders [17-19].

\section{Appendix C Bound state energies of the Yukawa potential}

We derive in this appendix a quasi-analytical form of the eigen energies of the Yukawa potential $\mathrm{V}(\mathrm{x})$ $=\mathrm{a} \exp (-\mathrm{b}|\mathrm{x}|)$. They are related to the order of those Bessel functions of the first kind that take a zero for 
a specific argument [10]. By linearizing this order around zero we are able to obtain the perturbative behavior of the ground state eigen energy in the limit of weak binding $\left(8 \mathrm{a} \mathrm{m} / \mathrm{b}^{2}<<1\right)$. We consider the Schrödinger equation, given in atomic units (with $\mathrm{h}=1$ ) as.

$$
\left[-1 /(2 \mathrm{~m}) \mathrm{d}^{2} / \mathrm{dr}^{2}-\mathrm{V}_{0} \exp (-\mathrm{b}|\mathrm{x}|)\right] \Psi(\mathrm{r})=\mathrm{E} \Psi(\mathrm{x})
$$

with parameters $\mathrm{V}_{0}>0$ and $\mathrm{b}>0$, eigenvalue $\mathrm{E}<0$ for bound states, and the corresponding eigen function $\Psi(\mathrm{x})$. We first rescale the coordinate $\mathrm{x}$ to a unitless position $\mathrm{t} \equiv \mathrm{x} \mathrm{b} / 2$, energy $\lambda \equiv\left(8 / \mathrm{b}^{2}\right) \mathrm{m} \mathrm{E}$ and effective potential strength $\alpha \equiv\left(8 \mathrm{~V}_{0} / \mathrm{b}^{2}\right) \mathrm{m}$. The rescaled equation $(\mathrm{C} 1)$ turns into

$$
\left[\mathrm{d}^{2} / \mathrm{dt}^{2}+\alpha \exp (-2|\mathrm{t}|)\right] \Psi(\mathrm{t})=-\lambda \Psi(\mathrm{t})
$$

Since our potential $\mathrm{V}(\mathrm{x})$ is an even function of $\mathrm{r}$, all eigenstates can be chosen with definite parity. As a result the wave function for half of the space $(t>0)$ provides sufficient information. Note that states with odd parity have to vanish at $\mathrm{t}=0$, while for even parity states the spatial derivative vanishes at $\mathrm{t}=0$. Bound states are spatially localized and therefore approach zero for $\mathrm{t} \rightarrow \pm \infty$. This boundary condition is crucial for the eigen values. We will come back to this a little later. As we are consider $t>0$, from now on we will remove the absolute value sign in the potential in (C2). Next we make the following transformation $\mathrm{t} \equiv$ $(1 / 2) \ln (\alpha)-\ln (\mathrm{z})$, or equivalently, $\mathrm{z} \equiv \exp (-\mathrm{t}) \sqrt{ } \alpha$. It is easy to show that $\mathrm{d} / \mathrm{dt}=-\mathrm{zd} / \mathrm{dz}, \mathrm{d}^{2} / \mathrm{dt}^{2}=\mathrm{zd} / \mathrm{dz}+$ $\mathrm{z}^{2} \mathrm{~d}^{2} / \mathrm{dz} \mathrm{z}^{2}$, and $\mathrm{z}^{2}=\alpha \exp (-2 \mathrm{t})$. For negative eigenvalues $\lambda$ we introduce $v^{2} \equiv-\lambda$ with $v>0$ such that Eq. (C2) turns into:

$$
\mathrm{z}^{2} \mathrm{~d}^{2} \Psi(\mathrm{z}) / \mathrm{dz} \mathrm{z}^{2}+\mathrm{zd} \Psi(\mathrm{z}) / \mathrm{dz}+\left(\mathrm{z}^{2}-\mathrm{v}^{2}\right) \Psi(\mathrm{z})=0
$$

The solution to equation (C3) can be expressed as a linear combination of Bessel functions of the first kind, i.e., $\Psi(\mathrm{z})=\mathrm{A} \mathrm{J}(v ; \mathrm{z})+\mathrm{B} \mathrm{J}(-\mathrm{v} ; \mathrm{z})$. The original limit $\mathrm{x} \rightarrow 0$ corresponds to $\mathrm{z} \rightarrow \sqrt{ } \alpha$ and $\mathrm{r} \rightarrow \infty$ is mapped to $\mathrm{z}$ $\rightarrow 0$, such that the bound states have to fulfill $\Psi(\mathrm{x}=\infty) \rightarrow 0$ requiring $\Psi(\mathrm{z}=0) \rightarrow 0$. As we assumed $v>0$ and since $\mathrm{J}(-\mathrm{v} ; \mathrm{z}=0)=\infty$, we have to assume that $\mathrm{B}=0$ and obtain $\Psi(\mathrm{z})=\mathrm{A} \mathrm{J}(v ; \mathrm{z})$ with normalization constant $\mathrm{A}$ to be determined.

In order to determine the possible values of $v$ we consider the even and odd parities separately. For 
even parity states, the condition $\mathrm{d} \Psi(\mathrm{t}=0) / \mathrm{dt}=0$ leads to the requirement $\mathrm{d} \Psi(\mathrm{z}) /\left.\mathrm{dz}\right|_{\mathrm{z}}=\sqrt{\alpha}=0$, where we have used $\mathrm{d} / \mathrm{dt}=-\mathrm{z} \mathrm{d} / \mathrm{dz}$, or equivalently

$$
\mathrm{d} J(v ; z) /\left.d z\right|_{z}=\sqrt{ } \alpha=0
$$

Very similarly, for the even parity states $[\Psi(\mathrm{t}=0)=0]$ leads to the constraint $\Psi(\mathrm{z}=\sqrt{\alpha})=0$ or equivalent

$$
\left.\mathrm{J}(\mathrm{v} ; \mathrm{z})\right|_{\mathrm{z}}=\sqrt{\alpha}=0
$$

Eqs. (C4) are interesting as in spectral analysis the eigenvalues are usually obtained as the roots of the arguments of functions and not necessarily of their order as in our case. The permitted discrete values of the order $v$, denoted by $\square v 0$, lead then to the desired exact bound state energies $\mathrm{E}$

$$
\mathrm{E}=-\mathrm{b}^{2} v 0^{2} /(8 \mathrm{~m})
$$

Next we will show that for small potential strength a the ground state eigenvalue equation $\partial \mathrm{J}(v ; \mathrm{z}) / \partial \mathrm{z}$ $\mathrm{z}_{\mathrm{z}}=\sqrt{\alpha}=0$ predicts that the parameter $\mathrm{v} \square$ grows quadratically with $\alpha$. To simplify the derivative, we use $\partial J(v ; z) / \partial z=[J(-1+v ; z)-J(1+v ; z)] / 2$ and abbreviate $f(v ; z) \equiv \partial J(v ; z) /\left.\partial z\right|_{z}=\sqrt{ } \alpha$. To find the root of $f(v ; z)$ in $v$, we first Taylor expand the function $f(v ; z)$ around $v=0$, leading to $f(v ; z)=f(0 ; z)+v \partial f(v ; z) /\left.\partial v\right|_{v=0}+O\left[v^{2}\right]$. Using this linearized form for $f(v ; z)$ we can solve for $v_{0}$ such that we have $f\left(v_{0} ; z\right)=0$. We obtain the solution $v_{0}=-\mathrm{f}(0 ; \mathrm{z}) /[\partial \mathrm{f}(0 ; \mathrm{z}) / \partial v]$. The numerator becomes $\mathrm{f}(0 ; \mathrm{z})=-\mathrm{J}(1 ; \mathrm{z})$, which for small $\mathrm{z}$ simplifies to $-\mathrm{J}(1 ; \mathrm{z})=-\mathrm{z} / 2+O\left[\mathrm{z}^{3}\right]$.

The denominator $\partial \mathrm{f}(0 ; \mathrm{z}) / \partial v=[\partial \mathrm{J}(-1+v ; \mathrm{z}) / \partial v-\mathrm{J}(1+v ; \mathrm{z}) / \partial v] /\left.2\right|_{v=0} \equiv\left[\mathrm{T}_{1}+\mathrm{T}_{2}\right] / 2$ is more complicated. To find useful closed expressions for the derivative of the Bessel function with respect to its order $v$ is nontrivial, see [20]. Using that for a general order $\mu$ we have

$$
\partial \mathrm{J}(\mu ; \mathrm{z}) / \partial \mu=\mathrm{J}(\mu ; \mathrm{z}) \ln (\mathrm{z} / 2)+(\mathrm{z} / 2)^{\mu} \Sigma_{\mathrm{m}=0}\left(-\mathrm{z}^{2} / 4\right)^{\mathrm{m}} / \mathrm{m} ! \mathrm{d}[1 / \Gamma(\mu+1+\mathrm{m})] / \mathrm{d} \mu
$$


where $\mathrm{d}[1 / \Gamma(\mu+1+\mathrm{m})] / \mathrm{d} \mu=-\mathrm{P} \Gamma[1+\mathrm{m}+\mu] / \Gamma[1+\mathrm{m}+\mu]$ and $\mathrm{P} \Gamma$ denotes the digamma function. More specifically, for $\mu=-1+v$ and $\mu=-1+v$ we obtain

$$
\begin{aligned}
& \mathrm{T}_{1}=\mathrm{J}(-1+\mathrm{v} ; \mathrm{z}) \ln (\mathrm{z} / 2)-(\mathrm{z} / 2)^{-1+v} \Sigma_{\mathrm{m}=0}\left(-\mathrm{z}^{2} / 4\right)^{\mathrm{m}} / \mathrm{m} ! \mathrm{P} \Gamma[\mathrm{m}+\mathrm{v}] / \Gamma[\mathrm{m}+\mathrm{v}] \\
& \mathrm{T}_{2}=\mathrm{A} 2 \equiv \mathrm{J}(1+\mathrm{v} ; \mathrm{z}) \ln (\mathrm{z} / 2)-(\mathrm{z} / 2)^{1+v} \Sigma_{\mathrm{m}=0}\left(-\mathrm{z}^{2} / 4\right)^{\mathrm{m}} / \mathrm{m} ! \text { РГ[2+m+v] } / \Gamma[2+\mathrm{m}+\mathrm{v}]
\end{aligned}
$$

For $v=0$ these two terms simplify to:

$$
\begin{aligned}
& \mathrm{T}_{1}=\mathrm{J}(-1 ; \mathrm{z}) \ln (\mathrm{z} / 2)-(\mathrm{z} / 2)^{-1} \sum_{\mathrm{m}=0}\left(-\mathrm{z}^{2} / 4\right)^{\mathrm{m}} / \mathrm{m} ! \mathrm{P} \Gamma[\mathrm{m}] / \Gamma[\mathrm{m}] \\
& \mathrm{T}_{2}=\mathrm{J}(1 ; \mathrm{z}) \ln (\mathrm{z} / 2)-(\mathrm{z} / 2)^{1} \sum_{\mathrm{m}=0}\left(-\mathrm{z}^{2} / 4\right)^{\mathrm{m}} / \mathrm{m} ! \mathrm{P} \Gamma[2+\mathrm{m}] / \Gamma[2+\mathrm{m}]
\end{aligned}
$$

Next, using the limits $\mathrm{z} \rightarrow 0$ and $\lim _{\mathrm{m} \rightarrow 0} \mathrm{P} \Gamma[\mathrm{m}] / \Gamma[\mathrm{m}]=-1$ we obtain $\mathrm{T}_{1} \rightarrow(\mathrm{z} / 2)^{-1}$ while $\mathrm{T}_{2} \rightarrow 0$. As our final result we have $[\partial J(-1+v ; z) / \partial v-J(1+v ; z) / \partial v] /\left.2\right|_{v=0}=1 / z$ for small values of $z$. Returning to $v_{0}=-$ $\mathrm{f}(0 ; \mathrm{z}) / \partial \mathrm{f}(0 ; \mathrm{z}) / \partial v$ this amounts to $v_{0}=\mathrm{z}^{2} / 2$. In other words, we obtain for the linearized form of the energy

$$
\mathrm{E}_{\operatorname{lin}}=-\left(2 \mathrm{~m} / \mathrm{b}^{2}\right) \mathrm{V}_{0}^{2}
$$

which is valid if the small dimensionless parameter $\left(8 \mathrm{~V}_{0} / \mathrm{b}^{2}\right) \mathrm{m}$ is less than unity.

\section{Appendix D Scaling of matrix elements with $\mathbf{L}$}

Here we compute the second-order contribution from perturbation theory for the limit $\mathrm{L} \rightarrow \infty$. In appendix B we derived that the second-order term is given by $\omega^{(2)}=\left\langle\omega^{(0)}|\mathrm{V}| \omega^{(1)}\right\rangle$. Using $\left|\omega^{(1)}\right\rangle=\mathrm{G}$ $\left(\mathrm{V}-\omega^{(1)}\right)\left|\omega^{(0)}\right\rangle$ and $\mathrm{G}\left|\omega^{(0)}\right\rangle=0$, we obtain $\omega^{(2)}=\left\langle\omega^{(0)}|\mathrm{V} \mathrm{G} \mathrm{V}| \omega^{(0)}\right\rangle$. Applied to our situation where the unperturbed energy $\omega^{(0)}$ is zero, we can express the resolvent in discrete momentum space as $\mathrm{G}=2 \mathrm{~m} \sum_{\mathrm{n}} \mathrm{k}_{\mathrm{n}}{ }^{-2}\left|\mathrm{k}_{\mathrm{n}}\right\rangle\left\langle\mathrm{k}_{\mathrm{n}}\right|$, where the discrete sum over the integers $\mathrm{n}$ excludes $\mathrm{n}=0$. Its action on a general state with the wave function $\Psi(\mathrm{x})$ can be expressed as $\mathrm{G} \Psi\left(\mathrm{x}^{\prime}\right)=(2 \mathrm{~m} / \mathrm{L}) \sum_{\mathrm{n}} \mathrm{k}_{\mathrm{n}}{ }^{-2} \exp (\mathrm{ik} \mathrm{n} \mathrm{x}) \int \mathrm{dx}{ }^{\prime} \exp \left(-\mathrm{ik}_{\mathrm{n}} \mathrm{x}^{\prime}\right)$ 
$\Psi\left(\mathrm{x}^{\prime}\right)$. As a result we obtain

$$
\begin{aligned}
\left\langle\omega^{(0)}\right| V & \text { G V }\left|\omega^{(0)}\right\rangle=\int d x L^{-1 / 2} V(x) G^{(0)} V\left(x^{\prime}\right) L^{-1 / 2} . \\
= & L^{-1} \int d x V(x)(2 m / L) \sum_{n} k_{n}^{-2} \exp \left(i k_{n} x\right) \int d x \exp \left(-i k_{n} x^{\prime}\right) V\left(x^{\prime}\right) . \\
= & m /\left(2 \pi^{2}\right) \int d x V(x) \sum_{n} n^{-2} \exp \left(i k_{n} x\right) \int d x^{\prime} \exp \left(-i k_{n} x^{\prime}\right) V\left(x^{\prime}\right) .
\end{aligned}
$$

where we have used $\mathrm{k}_{\mathrm{n}}=\mathrm{n} \Delta \mathrm{k}=\mathrm{n} 2 \pi / \mathrm{L}$. If we perform the limit $\mathrm{L} \rightarrow \infty$, and using that $\sum_{\mathrm{n}} \mathrm{n}^{-2}=\pi^{2} / 3$, the term simplifies to $\left\langle\omega^{(0)}|\mathrm{V} \mathrm{G} \mathrm{V}| \omega^{(0)}\right\rangle=\mathrm{m} / 6\left[\int \mathrm{dx} \mathrm{V}(\mathrm{x})\right]^{2}$ and therefore does not depend on the box size L.

A similar argument can also be applied to the higher-order terms that all increase with the box size L. 


\section{References}

[1] T. Kato, "Perturbation Theory for Linear Operators" (Springer-Verlag, Berlin, 1976).

[2] For a review, see, A. Messiah, "Quantum Mechanics” Vol II, (North-Holland, Amsterdam, 1966) and A. Galindo and P. Pascual, "Quantum Mechanics”, Vols. I and II (Springer Verlag, Berlin, 1990).

[3] C.M. Bender and S.A. Orszag, "Advanced Mathematical Methods for Scientists and Engineers: Asymptotic Methods and Perturbation Theory” (Springer, Berlin, 1999).

[4] J. M. Leinaas and E. Osnes, Phys. Scripta 22, 193 (1980).

[5] G.A. Arteca, F.M. Fernández and E.A. Castro, J. Math. Phys. 25, 2377 (1984).

[6] R. Ramaswamy and R.A. Marcus, J. Chem Phys. 74, 1379 (1981).

[7] E. Borel, Ann. Sci. École Norm. Sup. (3), 16, pp. 9-131 (1899).

[8] G.H. Hardy, "Divergent Series” (Clarendon Press, Oxford, 1949).

[9] Q.Z. Lv, D.J. Jennings, J. Betke, Q. Su and R. Grobe, Comp. Phys. Comm. 198, 31 (2016).

[10] See http://www.math.ru.nl/ koelink/edu/LM-dictaat-scattering.pdf and C.M. Fabre and D. Guery-Odelin, Am. J. Phys. 79, 755 (2011).

[11] O. Costin, "Asymptotics and Borel summability", CRC Monographs and Surveys in Pure and Applied Mathematics 141 (CRC Press 2008).

[12] G.A. Arteca, F.M. Fernández and E.A. Castro, "Large-Order Perturbation Theory and Summation Methods in Quantum Mechanics” (Springer-Verlag, Berlin, 1990).

[13] H. Grotch and D.A. Owen, Foundations of Physics 32, 1419 (2002).

[14] S. Weinberg, "The Quantum Theory of Fields, Volume 1: Foundations" (Cambridge University Press, Cambridge, 2005).

[15] E.E. Salpeter and H.A. Bethe, Phys. Rev. 84, 1232 (1951).

[16] B. Simon, Phys. Rev. Lett. 25, 1583 (1970).

[17] J. Paldus, Sec. 5 (page 76) in “Atomic, molecular and optical physics Handbook”, ed. G.F. Drake (AIP Press, Woodbury NY, 1996).

[18] B.R. Judd, J. Phys. C 14, 375 (1981).

[19] J.S. Bell, Nucl. Phys. 12, 117 (1959).

[20] J. Sesma arXiv:1401.4850v1 http://arxiv.org/abs/1401.4850v1 [math.CA, pdf: http://arxiv.org/pdf/1401.4850v1]. 